\title{
Instabilities in the excitation of non-radial pulsation modes in the $\delta$ Scuti star BV Circini ${ }^{\star}$
}

\begin{abstract}
L. Mantegazza ${ }^{\star \star}$
INAF - Osservatorio Astronomico di Brera, via E. Bianchi 46, 23807 Merate, Italy

Received 28 July 2003 / Accepted 21 October 2003

Abstract. High resolution spectroscopic observations obtained in 1995 of the $\delta$ Scuti star BV Circini are analyzed and the results compared with those obtained by Mantegazza et al. (2002) from spectroscopic observations in 1996 and 1998. Nonradial pulsation modes both of low and high degree have been observed. Most of them are the same detected in the other two campaigns, however the strongest high-degree one $\left(14.62 \mathrm{~cd}^{-1}\right)$ is different from that observed in $1996\left(13.85 \mathrm{~cd}^{-1}\right)$, which in turn is different from that observed in $1998\left(17.28 \mathrm{~cd}^{-1}\right)$. The $13.85 \mathrm{~cd}^{-1}$ mode is the only one of these independently detected in the three seasons, in which it kept about the same amplitude. One high-degree retrograde mode at $1.48 \mathrm{~cd}^{-1}$ has been independently detected in the three campaigns. The mode amplitude variations are discussed and it is shown that large variations can occur from one year to the next. The mode identifications performed on the three datasets by means of the fit of line profile variations supply results in good agreement among themselves. The high degree prograde modes have azimuthal orders between 12 and 14, while the retrograde one is about 7. Finally the stellar pulsation spectrum as derived from all the available spectroscopic and photometric data, and which consists of about 20 terms, is discussed.
\end{abstract}

Key words. methods: data analysis - techniques: spectroscopic - stars: individual: BV Cir - stars: oscillations stars: variables: $\delta$ Sct

\section{Introduction}

BV Circini ( $\equiv$ HD 132209) was discovered as a $\delta$ Scuti variable star by Kurtz (1981). Mantegazza et al. (2001; hereafter Paper I) performed and discussed simultaneous spectroscopic and photometric observations obtained in two campaigns in 1996 and 1998. Nine pulsation modes were detected from photometry and thirteen from spectroscopy; five modes are in common to both techniques. The spectroscopic data gave ample evidence of dramatic amplitude variations in some modes, in particular the strongest mode in 1998 was not detectable in 1996 data. The typing of the modes was performed by means of a simultaneous model fit of line profile and photometric variations. Among the results six modes were found to be prograde with azimuthal order ranging from -12 to -14 and one retrograde with $m \simeq 7$. The radial fundamental mode (the dominant one in the photometric data) was also observed $\left(6.33 \mathrm{~cd}^{-1}\right)$. The mode typing allowed the authors to estimate the inclination of the rotation axis to be about $60^{\circ}$, and, taking into account the derived value of $v \sin i=96 \pm 1 \mathrm{~km} \mathrm{~s}^{-1}$, an equatorial rotational velocity of $111 \pm 12 \mathrm{~km} \mathrm{~s}^{-1}$ was obtained. The pulsation analysis allowed also the authors to propose a rather accurate evaluation of the stellar physical parameters.

New observations of this star are useful to: a) give independent confirmation of the reality of the detected modes; b) allow

* Based on observations collected at ESO-La Silla.

$\star \star$ e-mail: luciano@merate.mi.astro.it better understanding of the extent of the mode instabilities and to get a more complete picture of the stellar pulsation spectrum.

Up to now there are few $\delta$ Scuti stars with a detailed study of line profile variations. For a list see Mantegazza (2000), to which 1 Mon (Balona et al. 2001) and QQ Tel (Koen et al. 2002) should be added.

\section{Spectroscopic observations and data processing}

The present observations were performed in the second part of the night during the spectroscopic campaign on the $\delta$ Scuti star FG Vir (Mantegazza \& Poretti 2002). The observations were performed during 5 consecutive nights (April 7-12,1995) with the Coudé Echelle Spectrograph (CES) attached to the $1.4 \mathrm{~m}$ Coudé Auxiliary Telescope (CAT) at the La Silla Observatory (ESO) via remote control from Garching. The CES was used with the short camera in the blue path configuration and a resolving power of 60000 set $(0.075 \AA$ of resolution). The spectrograms cover the range between 4490 and $4526 \AA$. 69 spectrograms with exposure times from 13 to 15 in were gathered in the 5 nights covering about 17 hours of observation (more than $3 \mathrm{~h}$ per night).

The spectra were flat fielded, rebinned into wavelengths and extracted using the ESO-MIDAS package. The resulting ASCII files were then cleaned of cosmic ray hits and normalized to the continuum and rebinned to heliocentric wavelengths by means of our codes. 


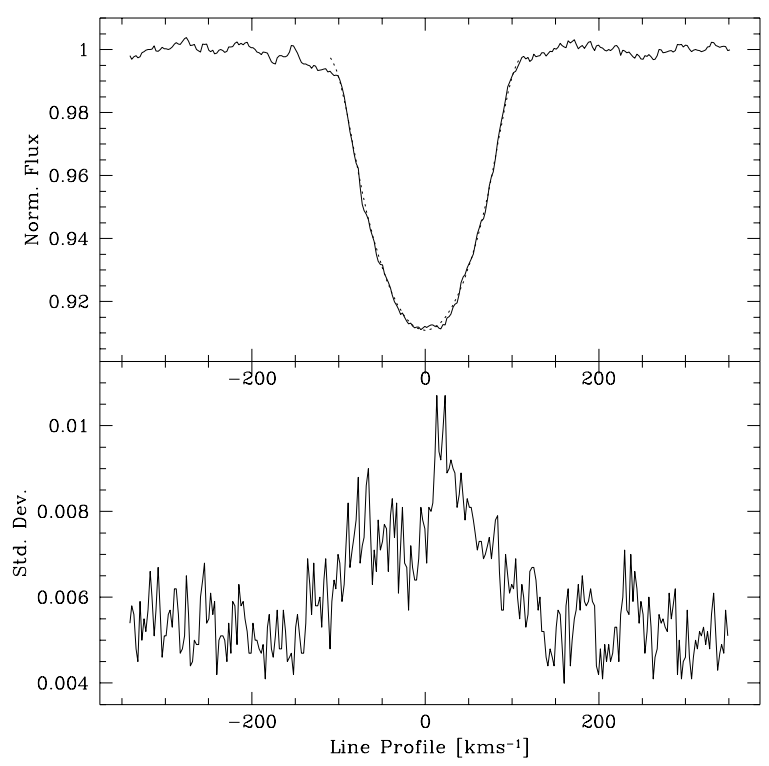

Fig. 1. Upper panel: profile of the FeII $4508 \AA$ line derived from the average of the all available spectrograms. The abscissae have been transformed from wavelengths to Doppler velocities with respect to the centre of the line. The dashed line shows the best fit profile derived from the convolution of an intrinsic profile with a rotationally broadened one with $v \sin i=97 \mathrm{~km} \mathrm{~s}^{-1}$. Lower panel: rms scatter about the mean of the flux in each individual pixel. The variability inside the line is evident.

Due to the relatively high projected rotation velocity, only the Fe II line at $4508.3 \AA$ is completely free of blends of adjacent features, and therefore it was the only one suitable for a study of line profile variations.

Figure 1 shows in the upper panel the average profile of this line, while in the panel below the standard deviation about the mean of each pixel is given. This panel shows clearly that there are variations in the line profile with a standard deviation up to about $0.5 \%$ of the local continuum intensity above the noise level.

The rms scatter of the pixels belonging to the continuum regions allows the estimation of the $S / N$ of the spectrograms. The resulting average value is 210 and the median is 212 .

A non-linear least-squares fit of a rotationally broadened Gaussian profile to the average line profile, considering also the limb darkening, allows us to get values very similar to those derived in the other two campaigns for the projected rotational velocity and the intrinsic width of the $4508 \AA$ line. We get $v \sin i=97.1 \mathrm{~km} \mathrm{~s}^{-1}$ and $W_{i}=12.0 \mathrm{~km} \mathrm{~s}^{-1}$. In Paper I the result was $v \sin i=96.5 \pm 1.0 \mathrm{~km} \mathrm{~s}^{-1}$ and $W_{i}=12.5 \pm 0.5 \mathrm{~km} \mathrm{~s}^{-1}$. The computed profile is shown as a dotted line in the upper panel of Fig. 1.

\section{Analysis of spectroscopic variations}

\subsection{Line profile moments}

Line profile moments are quantities, that, because they are integrated over the whole line profile (and hence the whole stellar disk), can be useful for the detection of low-degree modes (Mantegazza 2000).
The lower low-order moments were computed following the prescriptions by Balona (1986), and then their time series were frequency analyzed weighing the data according to their $S / N$. However due both to the unfavourable spectral window and because the data are rather noisy (we are working on a single line), useful results were obtained only for the first and second moments.

For the first moment (which is equivalent to the radial velocity of the line barycentre) we got that the data have a rms scatter of $1.9 \mathrm{~km} \mathrm{~s}^{-1}$ about the mean. The frequency analysis suggests the presence of two terms at $6.30 \mathrm{~cd}^{-1}$ and $8.02 \mathrm{~cd}^{-1}$ (or $7.02 \mathrm{~cd}^{-1}$ ) respectively. The first corresponds to the dominant photometric mode and had already been detected analyzing the spectrograms both of the 1996 and 1998 campaigns. In Paper I it was identified as the radial fundamental mode. The second term has not been previously detected. Both terms have about the same semi-amplitude of $1.0 \pm 0.3 \mathrm{~km} \mathrm{~s}^{-1}$. For the $6.30 \mathrm{~cd}^{-1}$ mode this value is not significantly different from the value of $1.3 \pm 0.1 \mathrm{~km} \mathrm{~s}^{-1}$ observed in both the other two seasons.

The frequency analysis of the second mode supplies only one significant term at $7.86 \mathrm{~cd}^{-1}$, a value that is not significantly different from the frequency of $7.89 \mathrm{~cd}^{-1}$ detected both in photometric data and in the two previous spectroscopic campaigns. Moreover this was also the only term detected in the second moment data of those campaigns. In Paper I it was identified as a low-degree non-radial mode with $\ell=1$ or 2 and $m=-1$ or -2 .

The fact that it is not detectable in the second moment variations confirms the axisymmetric nature of the $6.30 \mathrm{~cd}^{-1}$ mode, and suggests the same for the mode at $8.02 \mathrm{~cd}^{-1}$. The ratio between the frequencies of these two modes is 0.785 , close but consistently higher than the value expected between first and fundamental radial modes $\left(0.76<P_{0} / P_{1}<0.78\right.$, Breger 1989). Probably, if real, it should be a low-degree axisymmetric non-radial mode. For the moment, lacking other confirmations, we consider the detection of this mode as uncertain. It is desirable that the $8.02 \mathrm{~cd}^{-1}$ mode be independently detected in another dataset.

\subsection{Line profile variations (LPV)}

The variations of the shape of the line profiles was studied by a pixel by pixel analysis. A detailed description of this approach has been made by Mantegazza \& Poretti (1999) and by Mantegazza (2000). The least-squares global power spectra of the three seasons are shown in Fig. 2 where it is apparent that low, medium and high frequency signals (up to about $18 \mathrm{~cd}^{-1}$ ) contribute to the line profile variations. It is immediately apparent the the highest peak is different in each season, being $14.64 \mathrm{~cd}^{-1}$ in $1995,13.83 \mathrm{~cd}^{-1}$ in 1996 and $17.33 \mathrm{~cd}^{-1}$ in 1998. If we continue the analysis looking for other periodicities we find 4 modes already detected in Paper I, namely, in order of detection, $13.85,14.94,14.38$ and $1.47 \mathrm{~cd}^{-1}$. In the cases of 14.94 and $14.38 \mathrm{~cd}^{-1}$ the analysis slightly favours their $1 \mathrm{~cd}^{-1}$ aliases at 13.94 and $15.38 \mathrm{~cd}^{-1}$ respectively, but we adopted those values for consistency with the results of the 


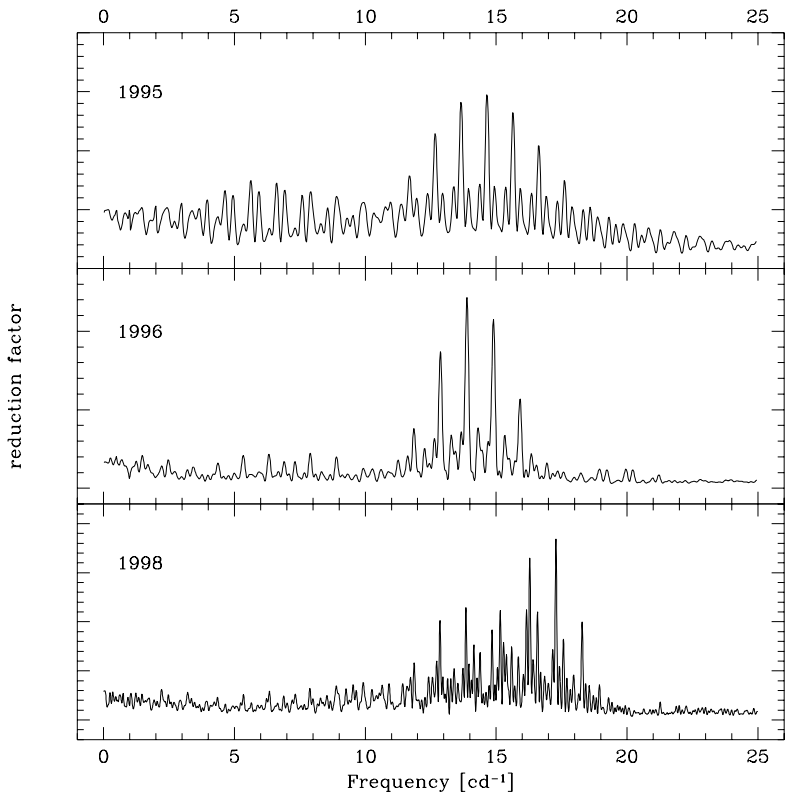

Fig. 2. Least-squares global power spectra of the LPV of the $4508 \AA$ line in the three observing seasons: the dominant peak is always different.

other two datasets. The residual spectrum, after introducing all these terms as "known constituents" (see Mantegazza 2000), shows peaks in the region below $8 \mathrm{~cd}^{-1}$, but does not allow any clear identification.

The behaviour of the phases of these terms across the line profile are shown in Fig. 3. The modes are arranged from top to bottom following the detection order and the phase datapoints are located by means of the corresponding formal error bars as derived from the least-squares fit. The $S / N$ ratio deteriorates going toward the lower amplitude modes (i.e. from top to bottom). The first four modes are progrades (phases decreasing with increasing Doppler velocity) and of comparable, relatively high order, while the last one $\left(1.47 \mathrm{~cd}^{-1}\right)$ is retrograde with a lower order.

\subsection{Mode identification}

It is possible to get estimates of the $\ell, m$ values of these modes by the non-linear least-squares fit of the line profile variations in the same way as in Paper I, with the technique described by Mantegazza (2000). To perform these fits we adopted the physical parameters derived in Paper I, in particular the inclination of rotational axis was fixed at $60^{\circ}$, as discussed in that paper. The phase curves derived from the best fitting sectoral modes are shown in Fig. 3 with the corresponding $\ell, m$ values written below the observed frequency of each mode. The uncertainties on the $\ell, m$ values are estimated to be of the order of unity.

If we compare the values derived here to those estimated in Paper I for the 4 modes in common (Paper I, Figs. 6 and 8), we find a very good agreement. This is summarized in Table 1 . In the last row we give also the $m$ identification of the $14.62 \mathrm{~cd}^{-1}$ mode, detected only in the 1995 data.

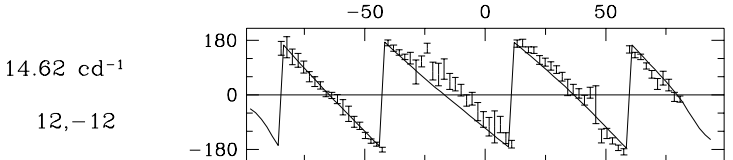

$13.85 \mathrm{~cd}^{-1}$

$13,-13$

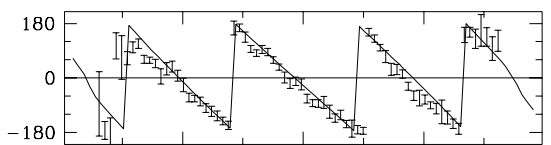

$14.94 \mathrm{~cd}^{-1}$

$13,-13$

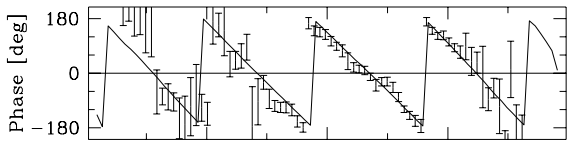

$14.38 \mathrm{~cd}^{-1}$

$14,-14$

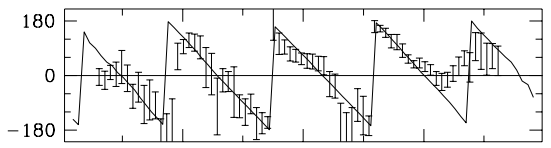

$1.47 \mathrm{~cd}^{-1}$

$7,+7$

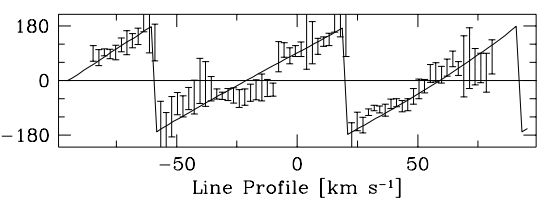

Fig. 3. Behaviour of the phases across the line profile of the 5 modes detected from LPV. The modes are arranged from top to bottom according to their order of detection. The solid lines show the values derived from the sectoral modes which best fit these phases; $\ell, m$ values are written below the corresponding frequency.

Table 1. $m$ values obtained from line profile variations in 3 independent datasets.

\begin{tabular}{rrrr}
\hline \hline \multirow{2}{*}{$\begin{array}{r}\text { Freq. } \\
{\left[\mathrm{cd}^{-1}\right]}\end{array}$} & \multicolumn{3}{c}{$m$} \\
\cline { 2 - 4 } 1.47 & 7 & 7 & 8 \\
13.85 & -13 & -12 & -13 \\
14.38 & -14 & -13 & -13 \\
14.94 & -13 & -15 & -13 \\
14.62 & -12 & - & - \\
\hline
\end{tabular}

\section{Amplitude variations of the modes}

To compare the different mode amplitudes and get estimates of their variability in different campaigns it would be useful to define and compute some significant numerical quantity. One possibility is to evaluate for each mode its power across the line profile, derived as the sum of the squared amplitudes at each line pixel as derived from the least-squares fit of a sine wave with the mode frequency. Unfortunately, for unequally spaced data the different frequency components in the Fourier space are no longer orthogonal, therefore the amplitudes derived in this way depend upon which modes and how many are assigned to the input model. In order to get an estimate of the mode amplitudes we performed the following procedure: line profile variations were fitted both with the model containing all the detected frequencies and with the models in which in turn one of these frequencies has been removed; some models also containing the dominant frequency in 1998 data, namely $17.28 \mathrm{~cd}^{-1}$, were also considered. For each mode the average of these estimates was then computed and the 
Table 2. Power across the line profile of the most significant highdegree modes in the three seasons.

\begin{tabular}{rrrr}
\hline \hline \multirow{3}{*}{$\begin{array}{r}\text { Freq. } \\
{\left[\mathrm{cd}^{-1}\right]}\end{array}$} & 1995 & 1996 & 1998 \\
\cline { 2 - 4 } 1.47 & $6.7 \pm 0.1$ & $3.1 \pm 0.2$ & $2.5 \pm 0.2$ \\
13.85 & $9.7 \pm 3.9$ & $10.8 \pm 4.3$ & $10.7 \pm 3.3$ \\
14.38 & $5.6 \pm 0.7$ & $7.3 \pm 2.6$ & $3.5 \pm 1.2$ \\
14.62 & $11.4 \pm 0.9$ & $2.9 \pm 0.9$ & $1.4 \pm 0.3$ \\
14.94 & $10.6 \pm 3.8$ & $7.5 \pm 2.6$ & $5.9 \pm 2.3$ \\
17.28 & $4.1 \pm 0.5$ & $1.2 \pm 0.2$ & $12.0 \pm 1.2$ \\
\hline detect. thres. & 4.8 & 3.0 & 2.0 \\
\hline
\end{tabular}

corresponding standard deviation was adopted as an indicator of the uncertainty on the result. Our result is shown in the second column of Table 2. The power is expressed in $10^{-4}$ squared units of the normalized continuum. The results regarding the modes at $1.47,14,38,14.62$, and $17.28 \mathrm{~cd}^{-1}$ are rather stable, their amplitudes not being appreciably affected by the other modes, while those of the modes at 13.85 and $14.94 \mathrm{~cd}^{-1}$ are unstable, because of their reciprocal interactions due to the shape of the spectral window.

The same process was applied to the 1996 and 1998 data and the results are reported in the two last columns of Table 2. By comparing the powers of the modes in the different seasons we can see that within the limits of its large uncertainties the $13.85 \mathrm{~cd}^{-1}$ mode was stable, and to some extent the same is valid for the $14.38 \mathrm{~cd}^{-1}$ mode. For the others there are large variations: for instance the excitation of the $1.47 \mathrm{~cd}^{-1}$ mode is decreasing with time, while the $17.28 \mathrm{~cd}^{-1}$, which was the strongest in 1998, had a much smaller amplitudes in 1995 and 1996. At the same time the $14.62 \mathrm{~cd}^{-1}$ (the strongest in 1995) had rather small amplitudes in 1996 and 1998.

This table allows us also to get an estimate of the lower threshold for an independent detection in each dataset. This can be obtained by comparing in each dataset the lowest amplitude of the independently detected terms with the highest one of the terms added to the fit, but not independently detected (i.e. $17.28 \mathrm{~cd}^{-1}$ for $1995 \mathrm{data}$, and $14.62 \mathrm{~cd}^{-1}$ for 1996 and 1998 data). These values are reported in the last row. We see that the detection threshold of the 1995 dataset is consistently higher than that of the others. These values allow us to understand why the medium frequency modes at 6.30 and $7.89 \mathrm{~cd}^{-1}$, detected from the moment analyses, have not been detected in this dataset by the pixel by pixel analysis: in Table 3 of Paper I we see that their amplitudes both in 1996 and in 1998 were below the present threshold, and from the moment analyses we see that their present amplitudes should be comparable to those.

\section{Discussion and conclusions}

The analysis of the 1995 spectroscopic observations of BV Cir gives a further independent confirmation of the reality of some of the modes detected in Paper I and moreover confirms that for some of these modes there are strong variations in their amplitudes passing from one year to the next. In contrast, the
Table 3. Summary of the modes detected in BV Cir.

\begin{tabular}{|c|c|c|c|c|c|c|}
\hline \multirow{2}{*}{$\begin{array}{l}\text { Freq. } \\
{\left[\mathrm{cd}^{-1}\right]}\end{array}$} & \multicolumn{3}{|c|}{ Spectr. } & \multicolumn{2}{|c|}{ Photom. } & \multirow[t]{2}{*}{ Commen } \\
\hline & 1995 & 1996 & 1998 & 1980 & 1998 & \\
\hline 0.65 & $\mathrm{~N}$ & $\mathrm{Y}$ & $\mathrm{Y}$ & $\mathrm{Y}$ & $Y$ & doubtful \\
\hline 1.47 & $\mathrm{Y}$ & $\mathrm{Y}$ & Y & $\mathrm{N}$ & $\mathrm{N}$ & HNRR \\
\hline 2.22 & $\mathrm{~N}$ & $\mathrm{~N}$ & $\mathrm{Y}$ & $\mathrm{N}$ & $\mathrm{N}$ & doubtful \\
\hline 6.33 & $\mathrm{Y}$ & Y & Y & $\mathrm{Y}$ & $\mathrm{Y}$ & $\mathrm{R}$ \\
\hline 7.89 & $\mathrm{Y}$ & Y & Y & $\mathrm{Y}$ & $\mathrm{Y}$ & LNR \\
\hline 8.02 & $\mathrm{Y}$ & $\mathrm{N}$ & $\mathrm{N}$ & $\mathrm{N}$ & $\mathrm{N}$ & doubtful \\
\hline 9.91 & $\mathrm{~N}$ & $\mathrm{~N}$ & $\mathrm{~N}$ & $\mathrm{Y}$ & $\mathrm{Y}$ & LNR \\
\hline 10.23 & $\mathrm{~N}$ & $\mathrm{~N}$ & $\mathrm{~N}$ & $\mathrm{Y}$ & $\mathrm{Y}$ & LNR \\
\hline 11.08 & $\mathrm{~N}$ & $\mathrm{~N}$ & $\mathrm{~N}$ & $\mathrm{Y}$ & $\mathrm{N}$ & doubtful \\
\hline 11.13 & $\mathrm{~N}$ & $\mathrm{~N}$ & $\mathrm{~N}$ & $\mathrm{Y}$ & $\mathrm{N}$ & LNR \\
\hline 11.63 & $\mathrm{~N}$ & Y & $\mathrm{Y}$ & $\mathrm{Y}$ & $\mathrm{Y}$ & LNR \\
\hline 12.29 & $\mathrm{~N}$ & $\mathrm{~N}$ & $\mathrm{~N}$ & $\mathrm{Y}$ & $\mathrm{Y}$ & LNR \\
\hline 12.38 & $\mathrm{~N}$ & Y & $\mathrm{N}$ & $\mathrm{Y}$ & $\mathrm{Y}$ & ?NR \\
\hline 13.85 & $\mathrm{Y}$ & $\mathrm{Y}$ & $\mathrm{Y}$ & $\mathrm{N}$ & $\mathrm{N}$ & HNRP \\
\hline 14.38 & $\mathrm{Y}$ & $\mathrm{Y}$ & $\mathrm{Y}$ & $\mathrm{N}$ & $\mathrm{N}$ & HNRP \\
\hline 14.62 & Y & $\mathrm{N}$ & $\mathrm{N}$ & $\mathrm{N}$ & $\mathrm{N}$ & HNRP \\
\hline 14.94 & $\mathrm{Y}$ & $\mathrm{Y}$ & $\mathrm{Y}$ & $\mathrm{N}$ & $\mathrm{N}$ & HNRP \\
\hline 15.80 & $\mathrm{~N}$ & $\mathrm{~N}$ & $\mathrm{Y}$ & $\mathrm{N}$ & $\mathrm{N}$ & HNRP \\
\hline 16.44 & $\mathrm{~N}$ & $\mathrm{~N}$ & $\mathrm{Y}$ & $\mathrm{N}$ & $\mathrm{N}$ & HNRP \\
\hline 17.28 & $\mathrm{~N}$ & $\mathrm{~N}$ & $\mathrm{Y}$ & $\mathrm{N}$ & $\mathrm{N}$ & HNRP \\
\hline
\end{tabular}

mode at $13.85 \mathrm{~cd}^{-1}$ (the strongest in 1996) seems to be quite stable.

The mode identification by fitting LPV confirms that there are several prograde non-radial modes with azimuthal order between 12 and 14 and one retrograde mode with $m \sim 7$. The derived values are in agreement, for the modes in common, with those obtained in Paper I.

In Table 3 all the modes detected up to now with the spectroscopic and photometric techniques are summarized.

To interpret this table it should be recalled that, due to the strong amplitude variations, for a mode to be real it is not necessary that it be detected in all datasets; the $S / N$ of its amplitude should be also considered. For example the modes at 14.62 and $17.28 \mathrm{~cd}^{-1}$ should be considered as real even if they were detected only in one dataset. The most uncertain modes are shown as "doubtful" in the last column of the table (see also Paper I). Looking at the Table we see that there are some modes detected both spectroscopically and photometrically; these are radial (R) or low-degree non radial modes (LNR); the least strong of these have been detected only photometrically, because of the better accuracy attainable with this technique. Among low frequency modes only that at $1.47 \mathrm{~cd}^{-1}$ was detected spectroscopically only because, as we have shown, it is a high-degree retrograde mode (HNRR). All the high frequency modes have been detected only spectroscopically because are high-degree prograde modes (HNRP). Thus in the stellar reference frame the frequencies of the pulsation modes span a narrower range that those in the observer reference frame and tend to clump in the region between about 6 and $12 \mathrm{~cd}^{-1}$. A similar result has been found by Kennelly et al. (1998) for $\tau$ Peg, and 
by Mantegazza (1997) for HD101158; moreover some preliminary results on $\epsilon$ Cep (Kennelly et al. 1998) and on V480 Tau (Hao et al., unpublished; see Di Mauro et al. 2002) seem to indicate the same behaviour. These are the $\delta$ Scuti stars with the highest $v \sin i$ values for which there is a detailed study of LPV. One could wonder if this could be a general result. However FG Vir, a star with low $v \sin i$ (but possibly with a low inclination, and hence a medium rotational velocity), has modes which span a much wider range of frequencies (Mantegazza \& Poretti 2002).

\section{References}

Balona, L. A. 1986, MNRAS, 220, 647

Balona, L. A., Bartlet, J. A. R., Caldwell, J., et al. 2001, MNRAS, 321, 239
Breger, M. 1989, DSS Newsletter, 1, 7

Di Mauro, M. P., Christensen-Dalsgaard, J., Pjipers, F., \& Hao, J. 2002, ASP Conf. Ser., 259, 336

Kennelly, E. J., Brown, T. M., Kotak, R., et al. ApJ, 495, 440

Kennelly, E. J., Brown, T. M., Ehrenfreund, P., et al. 1999, ASP Conf. Ser., 185, 284

Koen, C., Balona, L., van Wyk, F., et al. 2002, MNRAS, 330, 567

Kurtz, D. W. 1981, MNRAS, 196, 53

Mantegazza, L. 1997, A\&A, 323, 845

Mantegazza, L. 2000, in Delta Scuti and Related Stars, ed. M. Breger, \& M. H. Montgomery, ASP Conf. Ser., 210, 138

Mantegazza, L., \& Poretti, E. 1999, A\&A, 348, 139

Mantegazza, L., \& Poretti, E. 2002, A\&A, 396, 911

Mantegazza, L., Poretti, E., \& Zerbi, F. M. 2001, A\&A, 336, 547 (Paper I) 